\title{
Manifestaciones cutáneas de diabetes mellitus
}

\author{
Ximena Fajre ${ }^{1}$, Lilian Pérez², Javiera Pardoa, \\ Jorge D reysea, María Isabel Herane ${ }^{1}$.

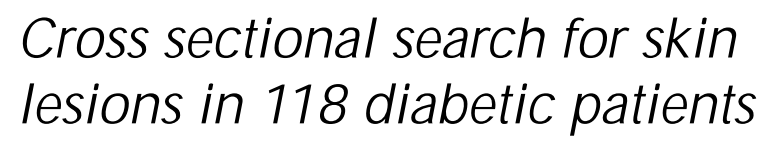

Background: Thirty percent of patients with diabetes mellitus have some skin lesion, that is directly related to the disease or caused by infections, complications or treatments used. Aim: To determine the frequency and type of skin lesions in diabetic patients. Material and methods: Cross sectional examination of 26 patients with type 1 diabetes, 75 patients with type 2 diabetes and 17 patients with gestational diabetes. A complete skin physical examination and a direct mycological examination and culture when onychomycosis was suspected, were performed. Results: In $56 \%$ of patients, diabetes lasted more than 10 years and $52 \%$ had some complication of the disease. A mean of $1.4 \pm 0.1$ skin lesions per patient was found among subjects with type 1 or 2 diabetes. No skin lesion was found in patients with gestational diabetes. The lesions found were onychomycosis in $27 \%$, diabetic dermatopathy in $17 \%$, macroangiopathy in 15\%, tinea corporis in $14 \%$ and xerosis in $14 \%$. Conclusions: Onychomycosis is the most commonly found skin lesion in diabetic patient (Rev Méd Chile 2009; 137: 894-9).

(Key w ords: Diabetes mellitus; Onychomycosis; Skin diseases)

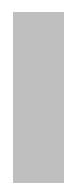

\begin{abstract}
Recibido el 26 de marzo, 2008. Aprobado el 30 de abril, 2009.
${ }^{1}$ Servicios de Dermatología, Hospital San Juan de Dios, Santiago de Chile y ${ }^{2}$ Hospital Félix Bulnes, Santiago de Chile.

anterno de Medicina. Facultad de Medicina, Pontificia Universidad Católica de Chile, Santiago de Chile.
\end{abstract}

L a diabetes mellitus (DM) es la más frecuente de las enfermedades metabólicas graves, estimándose su frecuencia en alrededor de $1 \%$ de la población general ${ }^{1}$. Corresponde a un grupo heterogéneo de alteraciones caracterizadas por un aumento en el nivel plasmático de glucosa y por alteraciones en el metabolismo de los carbohidratos y de los lípidos.

Según la clasificación vigente, hecha por el Comité Internacional de Expertos ${ }^{2,3}$ existen 4 cate-

Correspondencia a: Dra. Ximena Fajre W. Clínica AlemanaUniversidad del Desarrollo, Servicio de Dermatología. Av Vitacura 5951, Vitacura, Región Metropolitana, Chile. Fax: 7355479. E mail: xfajre@manquehue.net gonías de pacientes: 1) DM tipo 1; 2) DM tipo 2; 3 ) otros tipos específicos de diabetes, entre los que se encuentran defectos genéticos, endocrinopatías, enfermedades del páncreas exocrino y drogas, entre otros; 4) diabetes mellitus gestacional (DMG); identificándose además un 5o grupo donde se incluye intolerancia a la glucosa y glicemia de ayuno alterada, referidos a un estado metabólico intermedio entre la homeostasis normal de la glucosa y diabetes, lo que se denomina prediabetes.

El 90\% de los pacientes con DM tienen DM tipo 2. Se estima que alrededor de $30 \%$ de las personas con DM tienen algún tipo de compromiso cutáneo durante el curso de su enfermedad crónica ${ }^{1,4}$. 
El compromiso cutáneo de la DM se puede clasificar en cuatro categorías: 1) enfermedades cutáneas con fuerte y relativa asociación con DM; 2) infecciones cutáneas más frecuentes en este tipo de pacientes; 3) manifestaciones cutáneas de complicaciones diabéticas; 4) reacciones cutáneas relacionadas con el tratamiento antidiabético (Tabla 1).

Con el objeto de dilucidar la interrelación entre DM y piel se planteó un estudio de corte transversal con los siguientes objetivos: 1) Determinar la incidencia de manifestaciones cutáneas en un grupo de pacientes ambulatorios portadores de DM; 2) Determinar la eventual asociación entre el tipo de DM (DM tipo 1, DM tipo 2, DMG) y las lesiones cutáneas encontradas; 3) Determinar si algunas lesiones de piel pueden orientarnos fuertemente a la búsqueda de una DM en un paciente adulto.

\section{SUJETOS Y MÉTODO}

Se realizó un estudio de corte transversal en pacientes que concurren a control ambulatorio de diabetes del Servicio de Endocrinología y Diabetes del Hospital San Juan de Dios.

Se incluyeron pacientes portadores de DM tipo 1, DM tipo 2 y DMG en control. Se excluyeron 23 pacientes con diabetes descompensada, definida como hemoglobina glicosilada $>7 \%$ en los últimos 3 meses.

El estudio incluyó a 118 pacientes; fue realizado entre abril y diciembre de 2004 incorporándose previa aceptación de consentimiento informado, aprobado por el comité de ética de nuestra institución, a un protocolo donde se consignaron caractenísticas de edad, sexo, tipo de DM, tratamiento de DM, tiempo de evolución de DM y complicaciones asociadas.

\section{Tabla 1. M anifestaciones del compromiso cutáneo en pacientes con diabetes mellitus}

1. Enfermedades cutáneas asociadas a DM:

- Necrobiosis lipoidea diabeticorum (NLD)

- Granuloma anular

- Dermatopatía diabética

- Cambios esclerodermiformes de la piel

- Bulas diabéticas

- Piel amarilla

- Xantomas eruptivos

- Resistencia a insulina (Acantosis nigricans, obesidad, papilomatosis, lipodistrofias)

- Lesiones perforantes cutáneas

- Púrpuras pigmentarios

- Liquen plano oral, sensibilidades gingival, ardor, xerostomía

- Otros: S. de Alezzandrini, vitiligo, alopecia areata universalis

2. Infecciones cutáneas asociadas a DM:

- Candidiasis mucocutánea

- Infecciones bacterianas: eritrasma, estafilocócicas, estreptocócicas, otitis extrema por pseudomonas, infecciones por phycomycetes

- Dermatofitosis

3. Complicaciones cutáneas asociadas a DM:

- Macroangiopatía

- Microangiopatía

- Neuropatía (mal perforante plantar)

4. Reacciones cutáneas al tratamiento antidiabético:

- Reacciones alérgicas (sulfanilureas, clorpropamida, insulina)

- Lipoatrofía (inyecciones de insulina)

- Tumores insulínicos

- Queloides, atrofia dérmica focal, ampollas y pápulas hiperqueratósicas 
En el grupo de pacientes con DMG, el fundamento fisiopatológico tras este tipo de diabetes es distinto, ya que son expuestos a glicemias elevadas por un breve periodo de tiempo, el que es insuficiente para desarrollar manifestaciones cutáneas propias de dicha enfermedad, por lo que dentro del análisis de los resultados fueron incluidos en el grupo control.

Los pacientes fueron evaluados por uno de los autores (Fajre o Pérez) y se realizó: un examen físico de la piel, mucosas, cuero cabelludo y genitales. No se utilizaron otros métodos diagnósticos coadyuvantes (luz Wood, biopsia, etc.). En el grupo de pacientes en los que se sospechó onicomicosis o tiña corporis se realizó un examen micológico directo y cultivo de piel o fanéreos comprometidos.

Estadística. Se efectuó a través de métodos de asociación (chi cuadrado; Fisher; diferencia de proporciones; prueba del signo y prueba de McNemar) y pruebas de comparación de medias ( $t$ student; Mann Whitney; Wilcoxon; Anova; Bonferroni; Scheffé) utilizando los programas estadísticos EPINFO y STATA.

\section{Resultados}

De los 118 pacientes examinados, 66 (55,9\%) eran de sexo femenino y $52(44,1 \%)$ de sexo masculino. La edad promedio del grupo fue de 53,3 $\pm 19,7$ ( $\mathrm{x} \pm$ DS) años con un rango entre 8 y 89 años.

La distribución de los sujetos según el tipo de DM lo vemos en la Tabla 2. El 8,2\% de los pacientes se trataba sólo con dieta, 36,8\% necesitaba hipoglicemiantes orales (HGO) e insulina 55\%.

Tabla 2. D istribución de 118 pacientes según el tipo de diabetes

\begin{tabular}{|lrr|}
\hline Tipo diabetes & N & $\begin{array}{r}\text { \% de la } \\
\text { muestra }\end{array}$ \\
\hline Diabetes gestacional & 17 & 14,4 \\
Diabetes mellitus tipo 1 & 26 & 22,0 \\
Diabetes mellitus tipo 2 & 75 & 63,6 \\
\hline
\end{tabular}

El tiempo de evolución de la DM en 55,9\% era de más de 10 años.

En cuanto a las complicaciones asociadas a la DM (Tabla 3), 52,2\% del total presentó al menos alguna complicación al momento del estudio.

La distribución de las lesiones cutáneo-mucosas más frecuentes encontradas en los 118 pacientes examinados podemos resumirlos en la Figura 1.

$\mathrm{Si}$ dividimos las características de los diabéticos tipo 1 y tipo 2, vemos que de los 26 pacientes con DM tipo 1, 65,4\% (17) eran de sexo masculino. Este grupo tenía una edad promedio de 31,4 \pm 6,2 años. En 19,2\% hubo al menos una complicación asociada. En este grupo debemos considerar que 57,7\% tenía más de 10 años de evolución de su diabetes. Las complicaciones más frecuentemente asociadas a DM tipo 1 fueron nefropatía $15,4 \%$ (4), retinopatía $15,4 \%$ (4), angiopatía 7,7\% (2) y neuropatía $11,5 \%$ (3). En $11,5 \%$ (3) se pesquisó HTA. Las complicaciones cutáneo-mucosas más frecuentes en este grupo lo vemos en la Tabla 4. Hubo un total de 36 lesiones cutáneas lo que significa $1,4 \pm 0,27$ lesiones por paciente.

En el grupo de DM tipo 2, 41,3\% (31) era de sexo masculino. La edad promedio de este grupo fue de $65,2 \pm 7,5$ años. En 70\% hubo al menos alguna complicación asociada. En este grupo debemos considerar que $65,3 \%$ tenía una evolución de más de 10 años de su diabetes. Las complicaciones más frecuentemente en este grupo fueron retinopatía en $21,3 \%$ (16), neuropatía en $20 \%$ (15), angiopatía en $12 \%$ (9) y nefropatía en 9,3\% (7). En 33,3\% (25) se pesquisó HTA. Las complicaciones cutáneo-mucosas más frecuentemente encontradas en este grupo se resumen en la Tabla 5. Hubo un total de 107 lesiones cutáneas lo que significa $1,4 \pm 0,16$ lesiones por paciente.

\section{Tabla 3. Complicaciones asociadas a diabetes mellitus en 118 sujetos}

\begin{tabular}{|lrr|}
\hline Patología & N & \% \\
\hline Nefropatía & 12 & 10,8 \\
Retinopatía & 22 & 18,6 \\
Angiopatía & 11 & 9,3 \\
Neuropatía & 20 & 16,9 \\
Hipertensión arterial & 31 & 26,6 \\
\hline
\end{tabular}




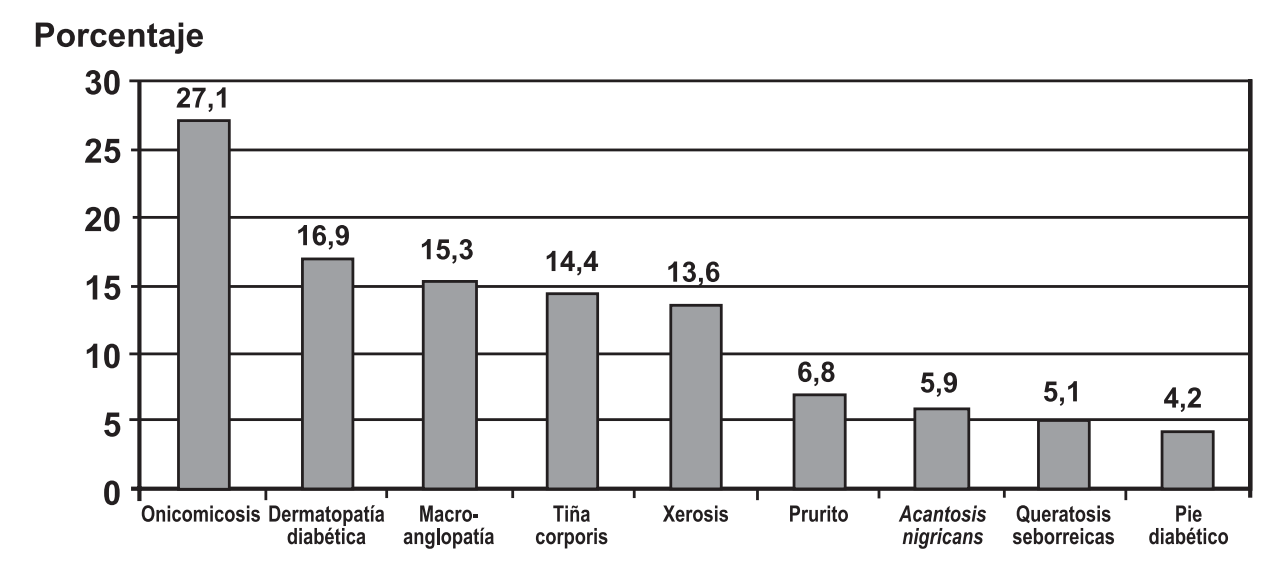

Figura 1. Distribución de lesiones cutáneo-mucosas más frecuentes ( $\mathrm{n}=140$ ).

Las lesiones cutáneo-mucosas más frecuentemente asociadas a DM tipo 1 y DM tipo 2, además de la xerosis en el primer grupo, fueron la onicomicosis, la dermatopatía y la macroangiopatía.

La dermatopatía diabética se caracteriza por áreas redondeadas u ovales de máculas brillantes generalmente atróficas muy frecuentemente bilaterales y asimétricas. Muchas veces estas lesiones eran secundarias a un traumatismo o a un fenómeno inflamatorio no bien precisado que dejaba una hiperpigmentación residual. El 60\% del grupo portador de dermatopatía era de sexo masculino con una edad promedio de 61,1 $\pm 13,6$ años. Cuatro pacientes (20\%) eran portadores de DM tipo 1 y 16 pacientes eran portadores de DM tipo 2. Estos pacientes en $75 \%$ presentaba al menos alguna complicación asociada, además de la dermatopatía.

La macroangiopatía caracterizada clínicamente como atrofia de piel de las piernas, palidez al elevarlas, frialdad de la piel y ortejos, distrofia ungueal, retraso en el retorno de la coloración postpresión se presentó en un grupo de 18 pacientes de los cuales $50 \%$ eran de sexo masculino, con edad promedio de 66,3 $\pm 15,6$ años. El $94,4 \%$ (17) de éstos tenían una DM tipo 2 y 5,6\% (1) una DM tipo 1.

En 83,3\% tenía una complicación asociada además de la macroangiopatía y en $50 \%$ hubo micológico directo positivo y $70 \%$ cultivo de hongos positivo de muestras examinadas de los pies.
Tabla 4. D istribución de lesiones cutáneo-mucosas más frecuentes en D M tipo 1

\begin{tabular}{|llr|}
\hline Lesión cutánea & N & $\%$ \\
\hline Xerosis & 8 & 32 \\
Tiña corporis & 8 & 32 \\
Onicomicosis & 4 & 16 \\
Dermatopatía diabética & 4 & 16 \\
Macroangiopatía & 1 & 4 \\
\hline
\end{tabular}

Tabla 5. D istribución de lesiones cutáneo-mucosas más frecuentes en D M tipo 2

\begin{tabular}{|lrr|}
\hline Lesión cutánea & N & \% \\
\hline Onicomicosis & 28 & 34,6 \\
Macroangiopatía & 17 & 21 \\
Dermatopatía diabética & 16 & 19,8 \\
Tiña corporis & 9 & 11,1 \\
Xerosis & 8 & 9,9 \\
Intertrigos & 1 & 1,2 \\
Foliculitis & 1 & 1,2 \\
Piodermias & 1 & 1,2 \\
\hline
\end{tabular}

La tercera lesión cutáneo-mucosa de mayor incidencia en el grupo investigado fue la onicomicosis. Esta estuvo presente en 32 pacientes y 
$43,8 \%$ eran hombres, con edad promedio de 66,7 $\pm 11,7$ años. El 87,5\% (28) eran portadores de DM tipo 2 y 12,5\% (4) de una DM tipo1. En 75\% de ellos se pesquisó al menos una complicación al momento del estudio. La distribución y análisis de los exámenes micológicos directo y cultivo son analizados en la Tabla 6.

Se observaron además, intertrigos, foliculitis y piodermias, pero en forma aislada, no estadísticamente significativo.

No se pesquisó patología dermatológica en el grupo de DMG ( $\mathrm{n}=17)$.

\section{Discusión}

La DM constituye la enfermedad metabólica más frecuente dentro de la población general ${ }^{1,4,5}$. Existe una amplia gama de manifestaciones cutáneo-mucosas asociadas a la DM que fluctúan en estimaciones de al menos $30 \%^{1}$ a valores de hasta $100 \%$ de afecciones dermatológicas en algún momento de la evolución de la enfermedad ${ }^{6,7}$.

En relación al tipo de DM el estudio inicialmente incluyó pacientes portadores de DM tipo 1, DM tipo 2 y DMG, sin embargo, en el análisis de resultados no se incluyó el grupo con DMG, debido a que la fisiopatología de este tipo de DM es distinta, siendo expuestos a hiperglicemias por un corto período de tiempo, lo que explicaría la ausencia de manifestaciones cutáneas propias de la DM.

En 101 pacientes estudiados se detectó un total de 1,4 $\pm 0,14$ lesiones cutáneas por paciente coincidiendo con las estimaciones de la literatura revisada, considerando sí que este estudio se hizo como corte transversal en un momento dentro de la evolución de la DM y en ausencia de un grupo control paralelo no portador de DM.

En el grupo de enfermos con DM tipo 1 y DM tipo 2 vemos que las lesiones cutáneo-mucosas más comúnmente observadas fueron: onicomicosis, dermatopatía y macroangiopatía diabética. Importante es considerar que en ambos grupos sobre $50 \%$ de las complicaciones asociadas ocurrieron en pacientes con DM de más de 10 años de evolución (57,7\% en portadores de DM tipo 1 y $65,3 \%$ en portadores de DM tipo 2).

La onicomicosis (al igual que las infecciones micóticas superficiales de la piel) puede ser la
Tabla 6. Exámenes micológicos directos y cultivos ( $n=30)$

\begin{tabular}{|lrr|}
\hline M icológico directo & N & $\%$ \\
\hline Levaduras & 2 & 6,7 \\
Dermatofitos & 14 & 46,7 \\
Levaduras y dermatofitos & 6 & 20 \\
\hline C ultivo hongos & $\mathbf{N}$ & $\%$ \\
\hline T rubrum & 19 & 63,3 \\
T Mentagrophytes & 4 & 13,3 \\
Cándida albicans & 2 & 6,7 \\
\hline
\end{tabular}

expresión de descompensación metabólica ${ }^{6-8}$ en nuestro estudio se presentó significativamente con mayor proporción en DM tipo 2 ( $p<0,001)$, en aquellos con complicaciones asociadas como HTA $(p<0,04)$ y en tratamiento con hipoglicemiantes orales $(p<0,03)$, planteándose una asociación entre el tipo de DM y la presencia de esta complicación.

La dermatopatía diabética presentó una frecuencia de $19,8 \%$ en el grupo total, sin encontrarse diferencia significativa entre los pacientes con DM tipo 1 y tipo 2 ( $p=0,58$ ). La macroangiopatía presentó una frecuencia de $17,8 \%$ en el grupo total, siendo significativamente más frecuente en el grupo de DM tipo 2 ( $p=0,037$ ).

Ambas complicaciones se describen en la literatura como manifestaciones cutáneas relacionadas con complicaciones degenerativas crónicas con frecuencias variables de $30 \%$ y $60 \%$ para la dermatopatía y de $30 \%$ para la macroangiopatía 9 . Nuestras cifras son cercanas a los valores de $20 \%$ descritos en la población general no diabéti$\mathrm{ca}^{10,11}$.

Considerando las afecciones dermatológicas no relacionadas con descompensación metabólica ni cambios degenerativos, detectamos prurito en $6,8 \%$, acantosis nigricans en $5,9 \%$ y queratosis seborreicas en $5,1 \%$. Lesiones específicas o predictivas de DM como necrobiosis lipoidea diabeticorum (NLD) no fueron detectadas debido a su baja incidencia $(0,3 \%)$ en estudios internacionales siendo sí un elemento de gran sospecha clínica (40\%$60 \%$ de portadores de NLD tienen una DM y en 
$15 \%$ de portadores de NLD predice la enfermedad con 2 años de anticipación) ${ }^{12}$.

Una afección dermatológica frecuente en los grupos estudiados fue la xerosis (15,8\%), que fue significativamente mayor en el grupo de DM tipo 1 ( $p=0,026)$. Su importancia estaría sujeta a la incidencia de xerosis en un grupo control ya que este signo clínico no aparece mencionado en la literatura revisada ${ }^{13,15}$.

La lipodistrofia secundaria al uso crónico de insulina, es una complicación frecuente en este subgrupo de pacientes, sin embargo no fue observada en nuestra muestra, debido probablemente a que los pacientes están entrenados para colocarse la insulina siempre en diferentes lugares.

En resumen, este corte transversal de 118 pacientes portadores de DM nos permite reconocer un porcentaje de $1,4 \pm 0,14$ lesiones por paciente con DM tipo 1 y DM tipo 2, e inferir que

\section{REFERENCIAS}

1. Pérez M, Steven K. Cutaneous manifestations of diabetes mellitus. J Am Acad Dermatol 1994; 30: 519-34.

2. The Expert Commttee on the Diagnosis and Classification of Diabetes MeLitus: Report of the Expert Committee on the Diagnosis and Classification of Diabetes Mellitus. Diabetes Care 1997; 20: 1118-97.

3. The Expert Committee on the Diagnosis and ClassificaTiON of Diabetes MeLuTus: Follow up report on the of the diagnosis of diabetes mellitus. Diabetes Care 2003; 26: 3160-7.

4. MuLER SA. Dermatologic disorders associated with diabetes mellitus. Mayo Clin Proc 1966; 41: 689-703.

5. Meurer M, Szeimies RM. Diabetes mellitus and skin diseases. Curr Probl 1991; 20: 11-23.

6. HunTLEY AC. The cutaneous manifestations of diabetes mellitus. J Am Acad Dermatol 1982; 7: 427-55.

7. Huntley AC. Cutaneous manifestations of diabetes mellitus. Dermatol Clin 1989; 7: 531-46.

8. Sibbald RG, Schachter RK. The skin and diabetes mellitus. Int J Dermatology 1984; 23: 567-84.

9. Ngo BT, Hayes KD, Di Miao DJ, Srinivasan SK, HuERTER CJ, RENDELL MS. Manifestations of cutaneous diabetic microangiopathy. Am J Clin Dermatol 2005; 6: 225-37. el examen de piel y mucosas en estos pacientes es necesario. Además la dermatopatía, macroangiopatía, xerosis y la onicomicosis estarían entre las lesiones cutáneo-mucosas más frecuentes, y existe un mayor compromiso de piel, mucosas y fanéreos generalmente en pacientes con más de 10 años de evolución de DM.

Seguramente, debido al bajo número de sujetos y al corto tiempo de exposición a hiperglicemias del grupo con DMG, no se logró pesquisar patologías dermatológicas en el presente estudio, sin embargo, sería interesante realizar un estudio multicéntrico en el cual se agrupase a un mayor número de sujetos con esta patología, y se realizara un seguimiento por un periodo de tiempo mayor al de la gestación, con el fin de correlacionar de mejor forma la presencia de patología dermatológica y el devenir de la diabetes mellitus gestacional.

10. BeRnsteIn JE. Cutaneous manifestations of diabetes mellitus. Curr Concepts Skin Dis 1980; 1: 3-10.

11. Rendell M, Bamisedun O. Diabetic cutaneous microangiopathy. Am J Med 1992; 93: 611-18.

12. FerRINGer T, MiLer F $3^{\text {RD }}$. Cutaneous manifestations of diabetes mellitus. Dermatol Clin 2002; 20: 483-92.

13. Richardson T, KeRR D. Skin-related complications of insulin therapy: epidemiology and emerging management strategies. Am J Clin Dermatol 2003; 4: 661-7.

14. NERN K. Dermatologic conditions associated with diabetes. Curr Diab Rep 2002; 2: 53-9.

15. Paron NG, LAmbert PW. Cutaneous manifestations of diabetes mellitus. Prim Care 2000; 27: 371-83.

16. Goodfield MJD, MiLaRd LG. The skin in diabetes mellitus. Diabetológica 1988; 31: 567-75.

17. GREENE RA, Scher RK. Nail changes associated with diabetes mellitus. J Am Acad Dermatol 1987; 16: 1015-21.

18. Lugo-Somolinos A, Sánchez JL. Prevalence of Dermatophytosis in patients with diabetes. J Am Acad Dermatol 1991; 26: 408-10.

19. HaRoon TS. Diabetes and skin: a review. Scott Med J 1974; 19: 257-67.

20. EDIDIN DV. Cutaneous manifestations of diabetes mellitus in children. Pedriatr Dermatol 1985; 2: 161-79. 